\title{
Impact of Performance Appraisal on Employee Motivation and Productivity in Turkish Forest Products Industry: A Structural Equation Modeling Analysis
}

\section{Utjecaj ocjene rada na motivaciju i produktivnost zaposlenika u turskoj industriji proizvoda na bazi šuma: analiza strukturnih jednadžbi modela}

Original scientific paper • Izvorni znanstveni rad

Received-prispjelo: 17. 2. 2017.

Accepted-prihvaćeno: 23. 5. 2018.

UDK: $630 * 79$

doi:10.5552/drind.2018.1710

\begin{abstract}
The present study investigates the influence of performance appraisal on employee motivation and productivity in Turkish forest products industry using a structural equation model. The questionnaires prepared for this purpose were applied to 432 people that work in 14 forest products industry businesses operating throughout Turkey. A total of nine hypotheses were established to determine the relationship between performance appraisal and employee motivation and productivity. The results indicated that five hypotheses were accepted, while four of them were rejected. In the light of the findings of this study, it may be generally said that the effect of performance appraisal on employee motivation and productivity was high. In other words, performance appraisal was found to be a major factor on employee motivation and productivity. The present study is expected to help managers and decision makers in selecting suitable motivating factors in order to retain and satisfy their employees. Consequently, this study will fill an important gap in the area of performance appraisal in the forest products industry by structural equation modeling and presents the opportunity for further studies.
\end{abstract}

Keywords: employee performance, forest product industry, organizational performance, structural equation modeling

SAŽETAK • Studija je prikaz istraživanja utjecaja ocjene rada na motivaciju i produktivnost zaposlenika u turskoj industriji proizvoda na bazi šuma primjenom modela strukturnih jednadžbi. Na upitnike pripremljene za tu namjenu odgovarale su 432 osobe zaposlene u 14 tvrtki u sektoru industrije na bazi šuma diljem Turske. Pritom

\footnotetext{
${ }^{1}$ Author is assistant professor at Karadeniz Technical University, Faculty of Forestry, Department of Forest Industry Engineering, Trabzon, Turkey. ${ }^{2}$ Author is lecturer at Karadeniz Technical University, Arsin Vocational School, Department of Material and Material Processing Technologies, Arsin/Trabzon, Turkey.

${ }^{1}$ Autor je docent Tehničkog sveučilišta u Karadenizu, Šumarski fakultet, Odjel za industrije bazirane na šumi, Trabzon, Turska. ${ }^{2}$ Autor je predavač Tehničkog sveučilišta u Karadenizu, Arsin škola, Odjel za procese obrade materijala, Arsin, Trabzon, Turska.
} 
je postavljeno devet hipoteza za utvrđivanje odnosa ocjene rada i motiviranosti i produktivnosti zaposlenika. Rezultati su pokazali da je pet hipoteza prihvatljivo, a četiri su odbijene. U svjetlu nalaza ove studije može se reći da je učinak ocjenjivanja na motiviranost zaposlenika i produktivnost vrlo visok. Drugim riječima, ocjena rada najviše utječe na motiviranost zaposlenika i na njihovu produktivnost. Očekuje se da će rezultati studije pomoći menadžerima i donositeljima odluka u odabiru odgovarajućih motivacijskih čimbenika kako bi zadržali i zadovoljili svoje zaposlenike. Stoga će ova studija ispuniti jaz koji postoji u području ocjenjivanja rada u drvnoj industriji modeliranjem strukturnih jednadžbi te će otvoriti mogućnosti daljnjeg istraživanja.

\section{Ključne riječi: uspješnost zaposlenika, drvna industrija, organizacija, modeliranje strukturnih jednadžbi}

\section{INTRODUCTION}

\section{UVOD}

Employee performance plays an important role in achieving organizational goals. In other words, employee performance is directly linked to the success of any organization. Therefore, organizations try to improve their productivity by providing an improvement in employee performance throughout the world. In this regard, various strategies have been developed to measure employee performance; one of them is performance appraisal (PA) (Wholey, 1999; Gichuhi et al., 2013).

The PA can be defined as a strategic and important approach that requires a regular inspection of the performance of employees in the organizations for performing the assigned tasks and responsibilities (Salau et al., 2014). This strategy aims to measure, assess and improve the performance of employees to achieve various objectives (Singh et al., 2010). The majority of organizations also use the PA approach to determine the factors such as salary increases, promotions, the need for individual development and training of employees (Gürbüz and Dikmenli, 2007). PA is thus accepted to be an important tool in order to improve both organizational performance and individual performance of employees. Such advantages of PA make it an important tool to reach organizational goals in many sectors (Vasset et al., 2011).

It is also important to emphasize that human capital (employees) in the organizations is considered as one of the most critical factors in gaining sustainable competitive advantage in the competitive situation of today's world. The organizations that have skilled and motivated employees may be regarded as advantageous in reaching such goals. Thus, many organizations endeavor to improve the human resource management and its functions (Chang and Han, 2006; Fakhimi and Raisy, 2013). In this respect, PA may be regarded as one of major functions of human resources managers.

PA intends to evaluate the performance of employees as objectively as possible. Kumbhar (2011) reported that a well-designed PA system helps develop employee performance-related criteria, provides a feedback mechanism and enables a more equitable reward system. It was also stated that employees' productivity increases when a fair system is available for PA in any organizations. In this regard, PA undertakes a critical task for organizations in order to reach their strategic objectives (Kumbhar, 2011). On the other hand, some researchers have pointed out that a rewarding system, applied as a result of PA, contributes to an increase in employee mo- tivation through influencing directly the performance of employees (Prowse and Prowse, 2009; Ochoti et al., 2012). Ali and Ahmed (2009) mentioned that rewards are managerial tools that help to reach organizational goals by affecting individual or group behavior. Therefore, most organizations use various types of rewards such as pay, promotion, bonuses in order to motivate employees and increase employees performance (Ali and Ahmed, 2009).

Furthermore, PA is a continuing procedure that measures expertise and achievements of employees based on an acceptable accuracy and equality. The employees are subjected to an appraisal process periodically after they are hired. The appraisal activities serve as a guide for managers in making a decision on employees' current job performance, awards, career goals and other job-related actions based on performance (Gürbüz and Dikmenli, 2007). However, the factors such as differences in the level of education, hiring temporary workers, employment, work experience, time pressures, and shift work might affect the appraisal process as well as employee motivation and productivity (Vasset et al., 2011).

Jelačić et al. (2008) found that the factors such as employment assurance, job organization and workplace activity affect employee motivation in forest products industry. In another study, no differences were found in terms of motivation factors among employees of Slovenian and Croatian wood industry companies. Besides, it was stated that managers should ensure the safety of employees and pay attention to their mutual relations (Kropivšek et al., 2011). Hitka and Štipalova (2011) compared the motivation of employees in the wood industry and other manufacturing enterprises for various categories throughout Slovakia. It was observed that the diversity of the importance of motivational factors is less significant in the category of employees. In another study on the impact of the economic crisis period on the motivation of forest products industry employees, it concluded that employee motivation does not differ in the period before and after the crisis (Hitka et al., 2014). Lorincova (2016) mentioned that increasing professional satisfaction of employees in the furniture industry may lead to an increase in motivation and business productivity.

In addition to the above mentioned investigations, a comprehensive analysis that considers the influence of PA on employee motivation and productivity is needed. Structural equation modeling (SEM) is an appropriate approach for this purpose. SEM is a versatile multivariate statistical approach that enables re- 


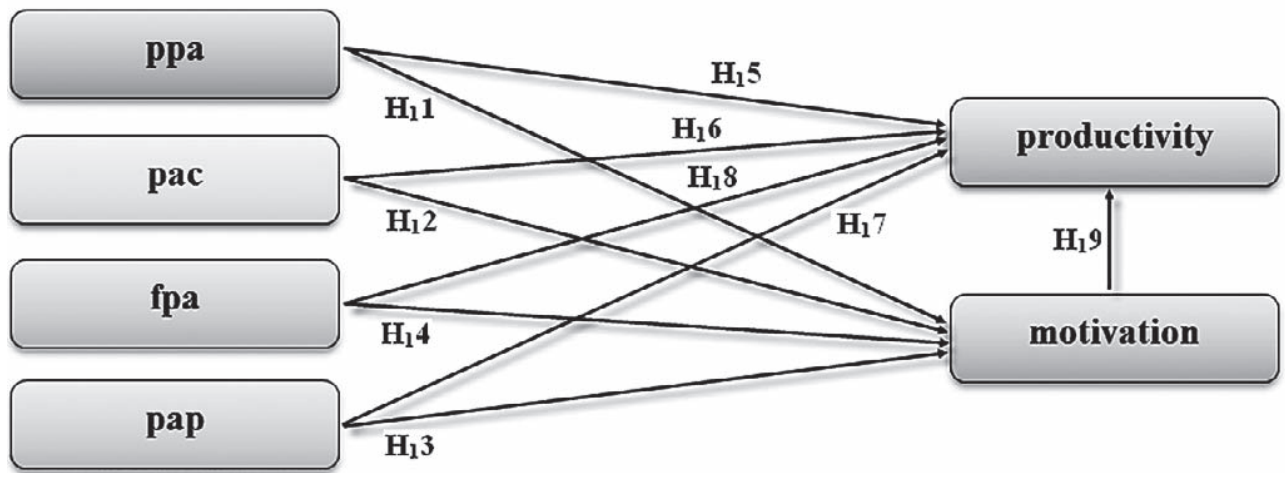

Figure 1 Conceptual model

Slika 1. Konceptualni model

searchers to effectively evaluate the relationships between observed and latent (unobserved) variables (Chan et al., 2007). The emergence of this approach has been regarded as a significant development and it has been widely employed in various scientific disciplines (Xiong et al., 2015). SEM is a combination of regression analysis, correlation, factor analysis, and path analysis. In comparison to other multivariate techniques, SEM is capable of estimating multiple and interrelated relationships and representing unobserved concepts in these relationships. This method also considers measurement errors in estimation. Further, it defines a model explaining an entire set of relationships (Xiong et al., 2014). Because of these advantages, SEM has been increasingly employed in many disciplines, such as banking industry (Osibanjo et al., 2014), education sector (Phin, 2015), service sector (Suki, 2014), business safety (Hsu et al., 2012), tourism sector (Hallak et al., 2012), food industry (Booth et al., 2013), and health sector (Bazarganipour et al., 2013), for uncovering the relationships among different kinds of variables.

\subsection{Objectives and hypotheses \\ 1.1. Ciljevi i hipoteze}

Although there are many applications to examine the effect of PA on motivation and productivity in various disciplines, limited information is available to investigate the influence of PA on employee motivation and productivity in forest products industry by the SEM. This study, therefore, analyses the relationships among various factors that affect motivation and productivity of employees of Turkish forest products industry by means of SEM to create a model of factors that both directly and indirectly affect employee motivation and productivity.

Additional objectives of this study were:

- to detect the factors that increase employee motivation and productivity;

- to investigate the relationship between employee motivation and productivity;

- to assess the effect of the PA on the motivation and productivity of employees.

In this regard, the following hypotheses were established based on independent and dependent variables.
$\mathrm{H}_{1} 1,2,3,4$ : There is a relationship between employee motivation and the purpose of PA (ppa), PA criteria (pac), PA practices (pap), Feedback in PA (fpa);

$\mathrm{H}_{1} 5,6,7,8$ : There is a relationship between employee productivity and the purpose of PA (ppa), PA criteria (pac), PA practices (pap), Feedback in PA (fpa);

$\mathrm{H}_{1}$ 9: There is a relationship between employee motivation and employee productivity.

In this perspective, we proposed the model composed of all the hypotheses that describe the relationships between two variables, as depicted in Figure 1.

From the model in Fig. 1, it can be seen that the purpose of the PA (ppa), PA criteria (pac), feedback in PA (fpa), and PA practices (pap) are considered as independent variables.

\section{MATERIALS AND METHODS} 2. MATERIJALI I METODE

\subsection{Population and sample}

2.1. Populacija i uzorak

The participants of the present investigation are composed of businesses that have ISO 9001:2008 Quality Management System (QMS) certifications and operate in forest products industry throughout Turkey. A previous study conducted by Serin (2004) has revealed that businesses that employ more than 100 employees give more significance to total quality management applications because they have a more institutional structure. Fourteen companies that meet these criteria agreed to implement the study. The survey was conducted between March 2015 and December 2015.

Among the 14 participating businesses, 7 deal with furniture, 6 with board products and 1 with paper. Surveys were administered to 432 people, including senior, middle and lower level employees.

\subsection{Data collection instruments}

\subsection{Način prikupljanja podataka}

A questionnaire prepared by compiling data from the surveys found in the literature was used in this study (Tarlı̆g, 2006; Y1lmaz, 2006). The survey is comprised of two sections. The first section contains 7 questions related to personnel information, while the other section includes Likert-type (5 scales) 51 questions related to the PA, employee motivation and employee productivity. 
After the surveys were completed, the data obtained were entered into SPSS (Statistical Software for Social Sciences). Analyses for reliability and validity were then performed by AMOS (Analysis of Moment Structures) package program.

\section{RESULTS AND DISCUSSION}

\section{REZULTATI I RASPRAVA}

\subsection{Demographic characteristics}

3.1. Demografska obilježja ispitanika

In this section, demographic information of the participants such as age, education, gender, marital status, wages, working experience and position in the company were determined. Results regarding demographic information for participant employees are given in Table 1.

Table 1 shows that $49.4 \%$ of the participants had vocational school and higher education levels. The majority of participants ( $82 \%$ ) were male, while $70.4 \%$ were married. In addition, $43.8 \%$ of the participants were employees, $49.1 \%$ of them had 6 years and over of working experience.

\subsection{Validity and reliability}

For the present study, Cronbach alpha coefficient was used for reliability analysis. According to the results of the analysis conducted, general Cronbach alpha coefficient was found to be 0.950 . This coefficient varies within a range of 0 to 1 , and a value over 0.90 means an excellent agreement (Kalayc1, 2009).

Moreover, factor analysis was carried out to measure structural validity. However, it is important to state that conformity of the data set for the factor analysis is tested with Kaiser-Meyer-Olkin (KMO) measure of sampling adequacy prior to the analysis. KMO should be over 0.5 for validity (Sharma, 1996). This value was found to be 0.917 for this study (Bartlett's Test of Sphericity Sig.:0.001). Thus, it can be said that the study was suitable for performing factor analysis. In the application of the factor analysis, varimax orthogonal rotation method and basic components analysis were considered. In this respect, factors that include less than three variables and variables that load on more than one factor were discarded. Furthermore, eigenvalues statistics was used in order to determine the number of factors. Dunteman (1989) reported that factors with an eigenvalue higher than 1 are considered significant. Table 2 presents the results of exploratory factor analysis related to the scale.

Upon application of factor analysis, from the scale consisting of 51 items, 18 items that do not match the structure of the scale or load on more than one factor were discarded. The remaining 33 items formed a structure that consists of 6 sub-factors with eigenvalues higher than 1. Total expressiveness of this six-factored structure was found to be $59.942 \%$.

\subsection{Measurement model}

3.3. Model mjerenja

Measurement model structure obtained as a result of factor analysis was tested by confirmatory factor analysis for investigating the structure validity. In order to test the conformity of the model, confirmatory factor analysis was performed by the AMOS. Standard prediction values, $t$ values and reliability level of the variables in the model are given in Table 3 .

In analyzing the standard prediction values of the variables, it was shown that the values vary between 0.491 and 0.846 . Besides, the $t$ values of these predic-

Table 1 Demographic information

Tablica 1. Demografski podatci

\begin{tabular}{|c|c|c|c|c|c|c|c|}
\hline \multicolumn{2}{|r|}{$\begin{array}{c}\text { Demographic variables } \\
\text { Demografske varijable }\end{array}$} & \multirow{2}{*}{$\begin{array}{l}N \\
4\end{array}$} & \multirow{2}{*}{$\begin{array}{c}\% \\
0.9\end{array}$} & \multicolumn{2}{|c|}{$\begin{array}{l}\text { Demographic variables } \\
\text { Demografske varijable }\end{array}$} & $N$ & $\%$ \\
\hline \multirow{6}{*}{$\begin{array}{c}\text { Educational } \\
\text { status } \\
\text { Obrazovanje }\end{array}$} & Literate / pismen & & & \multirow{6}{*}{$\begin{array}{l}\text { Salary } \\
\text { (TL) } \\
\text { Plaća }\end{array}$} & & & \\
\hline & Primary education / osnovno obrazovanje & 69 & 16.0 & & $\leq 999$ & 58 & 13.4 \\
\hline & High school / srednje obrazovanje & 146 & 33.8 & & $1000-1.499$ & 165 & 38.2 \\
\hline & Vocational school / stručno obrazovanje & 59 & 13.7 & & $1.500-1.999$ & 87 & 20.1 \\
\hline & University / visoko obrazovanje & 149 & 34.5 & & $2.000-2.499$ & 65 & 13.0 \\
\hline & Postgraduate / poslijediplomsko obrazovanje & 5 & 1.2 & & $\geq 2.500$ & 56 & 13.0 \\
\hline \multicolumn{2}{|l|}{ Total / Ukupno } & 432 & 100 & \multicolumn{2}{|l|}{ Total / Ukupno } & 431 & 99.8 \\
\hline \multirow{2}{*}{$\begin{array}{c}\text { Gender } \\
\text { Spol }\end{array}$} & Male / muški & 357 & 82.7 & \multirow{7}{*}{$\begin{array}{l}\text { Age } \\
D o b\end{array}$} & $16-19$ & 7 & 1.6 \\
\hline & Female / ženski & 71 & 16.4 & & $20-24$ & 46 & 10.6 \\
\hline \multicolumn{2}{|l|}{ Total / Ukupno } & 428 & 99.1 & & $25-29$ & 103 & 23.8 \\
\hline \multirow{4}{*}{$\begin{array}{l}\text { Working } \\
\text { experience } \\
\text { Radni staž }\end{array}$} & $0-5$ & 185 & 42.8 & & $30-34$ & 111 & 25.7 \\
\hline & $6-10$ & 143 & 33.1 & & $35-39$ & 93 & 21.5 \\
\hline & $11-20$ & 58 & 13.4 & & $40-49$ & 67 & 15.5 \\
\hline & $\geq 21$ & 11 & 2.5 & & $\geq 50$ & 5 & 1.2 \\
\hline \multicolumn{2}{|l|}{ Total / Ukupno } & 397 & 91,9 & \multicolumn{2}{|c|}{ Total / Ukupno } & 432 & 100 \\
\hline \multirow{3}{*}{$\begin{array}{l}\text { Position } \\
\text { Pozicija }\end{array}$} & Upper level / viša razina & 38 & 8.8 & \multirow{3}{*}{$\begin{array}{c}\text { Marital } \\
\text { status } \\
\text { Bračni status }\end{array}$} & $\begin{array}{c}\text { Married } \\
\text { oženjen/udana }\end{array}$ & 304 & 70.4 \\
\hline & Middle level / srednja razina & 175 & 40.5 & & Single / samac & 119 & 27.5 \\
\hline & Worker / radnik & 189 & 43.8 & & Other / ostalo & 1 & 0.2 \\
\hline \multicolumn{2}{|r|}{ Total / Ukupno } & 402 & 93.1 & \multicolumn{2}{|c|}{ Total / Ukupno } & 424 & 98.1 \\
\hline
\end{tabular}


Table 2 Results of scale's exploratory factor analysis

Tablica 2. Rezultati faktorske analize

\begin{tabular}{|c|c|c|c|c|c|}
\hline & $\begin{array}{l}\text { Variables } \\
\text { Varijable }\end{array}$ & $\begin{array}{l}\text { Factor } \\
\text { load } \\
\text { Težina } \\
\text { čimbenika }\end{array}$ & $\begin{array}{l}\text { Eigen } \\
\text { value } \\
\text { Vlastita } \\
\text { vrijednost }\end{array}$ & $\begin{array}{c}\text { Announced } \\
\text { variance } \\
\text { Predvidena } \\
\text { varijanca } \\
\% \\
\end{array}$ & $\begin{array}{l}\text { Cronbach's } \\
\text { Alpha } \\
\text { Cronbachov } \\
\text { alfa }\end{array}$ \\
\hline & PA purpose / Svrha ocjene rada & & 4.358 & 13.201 & 0.873 \\
\hline q3 & $\begin{array}{l}\text { In PA studies, the employees' ability for own business } \\
\text { regulation and planning is measured / Pri ocjeni rada mjerena } \\
\text { je sposobnost zaposlenika za samostalno reguliranje } i \\
\text { planiranje poslovanja. }\end{array}$ & 0.73 & & & \\
\hline $\mathrm{q} 2$ & $\begin{array}{l}\text { In PA studies, the employees' ability to make decisions are } \\
\text { measured / Pri ocjeni rada mjerena je sposobnost zaposlenika } \\
\text { da donose odluke. }\end{array}$ & 0.73 & & & \\
\hline $\mathrm{q} 1$ & $\begin{array}{l}\text { In PA studies, the employees' job information is measured } \\
\text { Pri ocjeni rada mjerena je informiranost zaposlenika o poslu. }\end{array}$ & 0.71 & & & \\
\hline $\mathrm{q} 4$ & $\begin{array}{l}\text { In PA studies, the employees' labor and the ability to use } \\
\text { correctly the resources are measured / Pri ocjeni rada mjeren je } \\
\text { rad zaposlenika i sposobnost ispravne uporabe resursa. }\end{array}$ & 0.69 & & & \\
\hline q5 & $\begin{array}{l}\text { In PA studies, the employees' ability to communicate effectively } \\
\text { is measured / Pri ocjeni rada mjerena je sposobnost zaposlenika } \\
\text { da učinkovito komuniciraju. }\end{array}$ & 0.69 & & & \\
\hline q6 & $\begin{array}{l}\text { In PA studies, the employees' cooperation understanding is } \\
\text { measured / Pri ocjeni rada mjereno je razumijevanje suradnje } \\
\text { zaposlenika. }\end{array}$ & 0.64 & & & \\
\hline q7 & $\begin{array}{l}\text { In PA studies, the employees' harmony with the environment } \\
\text { and respectful behavior are measured / Pri ocjeni rada mjerena } \\
\text { je usklađenost zaposlenika s okolinom i njegovo ponašanje. }\end{array}$ & 0.63 & & & \\
\hline & PA criteria / Kriteriji ocjene rada & & 3.715 & 11.258 & 0.854 \\
\hline q26 & $\begin{array}{l}\text { My PA score significantly affects my salary. / Ocjena moga } \\
\text { rada znatno utječe na moju plaću. }\end{array}$ & 0.66 & & & \\
\hline q27 & $\begin{array}{l}\text { My PA score significantly affects my promotion / Ocjena moga } \\
\text { rada znatno utječe na moje promaknuće. }\end{array}$ & 0.64 & & & \\
\hline q16 & $\begin{array}{l}\text { PA system used in our business is generally sufficient. / Sustav } \\
\text { ocjenivanja rada koji se primjenjuje u našem poslovanju } \\
\text { općenito je dovoljan. }\end{array}$ & 0.63 & & & \\
\hline q18 & $\begin{array}{l}\text { PA system used in our business is able to distinguish really } \\
\text { successful staff from unsuccessful staff / Sustavom ocjenivanja } \\
\text { rada koji se primjenjuje u našem poslovanju moguć je } \\
\text { razlikovati stvarno uspješne zaposlenike od neuspješnih. }\end{array}$ & 0.59 & & & \\
\hline q17 & $\begin{array}{l}\text { Getting high or low scores from PA is actually related to being } \\
\text { successful or unsuccessful. / Dobivanje visokih ili niskih ocjena } \\
\text { rada stvarno je povezano s uspjehom ili neuspjehom. }\end{array}$ & 0.57 & & & \\
\hline q25 & $\begin{array}{l}\text { I need to work hard to go beyond a certain score in PA / Moram } \\
\text { naporno raditi kako bih postigao određene rezultate pri } \\
\text { ocjenjivanju rada. }\end{array}$ & 0.56 & & & \\
\hline q20 & $\begin{array}{l}\text { My manager gives me a full score if I obtain an outstanding } \\
\text { achievement in my work / Moj menadžer daje mi najbolje } \\
\text { ocjene ako postignem izvrsne rezultate u radu. }\end{array}$ & 0.56 & & & \\
\hline q14 & $\begin{array}{l}\text { PA criteria include necessary factors for me to succeed in my } \\
\text { work. / Kriteriji ocjenjivanja obuhvaćaju čimbenike potrebne } \\
\text { za uspjeh u mom poslu. }\end{array}$ & 0.52 & & & \\
\hline & Feedback in PA / Povratne informacije o ocjeni rada & & 3.689 & 11.178 & 0.878 \\
\hline q33 & $\begin{array}{l}\text { In PA meeting, my manager tells me in what I am good / Na } \\
\text { sastanku vezanome za ocjenu moga rada menadžer mi kaže u } \\
\text { čemu sam dobar. }\end{array}$ & 0.73 & & & \\
\hline $\mathrm{q} 32$ & $\begin{array}{l}\text { In PA interview, my manager clearly points to what I am } \\
\text { missing. / Na sastanku vezanome za ocjenu moga rada } \\
\text { menadžer mi jasno kaže koji su moji nedostatci. }\end{array}$ & 0.73 & & & \\
\hline q35 & $\begin{array}{l}\text { In PA interview, my manager tells me my mistakes and failures } \\
\text { Na sastanku vezanome za ocjenu moga rada menadžer mi } \\
\text { priopći koje su moje pogreške i konkretni nedostatci. }\end{array}$ & 0.73 & & & \\
\hline
\end{tabular}


Table 2 Continue

Tablica 2. Nastavak

\begin{tabular}{|c|c|c|c|c|c|}
\hline & $\begin{array}{l}\text { Variables } \\
\text { Varijable }\end{array}$ & $\begin{array}{l}\text { Factor } \\
\text { load } \\
\text { Težina } \\
\text { čimbenika }\end{array}$ & $\begin{array}{l}\text { Eigen } \\
\text { value } \\
\text { Vlastita } \\
\text { vrijednost }\end{array}$ & $\begin{array}{l}\text { Announced } \\
\text { variance } \\
\text { Predvidena } \\
\text { varijanca } \\
\quad \% \\
\end{array}$ & $\begin{array}{l}\text { Cronbach's } \\
\text { Alpha } \\
\text { Cronbachov } \\
\text { alfa }\end{array}$ \\
\hline $\mathrm{q} 36$ & $\begin{array}{l}\text { In PA interview, my manager discusses with me about my } \\
\text { mistakes I cannot correct. / Na sastanku vezanome za ocjenu } \\
\text { moga rada menadžer razgovara sa mnom o mojim pogreškama } \\
\text { za koje ne znam kako ih ispraviti. }\end{array}$ & 0.70 & & & \\
\hline $\mathrm{q} 34$ & $\begin{array}{l}\text { In PA interview, my manager gives me the opportunity to express } \\
\text { my ideas clearly. / Na sastanku vezanome za ocjenu moga rada } \\
\text { menadžer mi daje mogućnost da jasno izrazim svoje ideje. }\end{array}$ & 0.68 & & & \\
\hline \multirow[t]{2}{*}{$\mathrm{q} 40$} & $\begin{array}{l}\text { In PA interview, I identify common goals with my manager } \\
\text { determining what I should do in future. / Na sastanku vezanome } \\
\text { za ocjenu moga rada zajedno sa svojim menadžerom identifici- } \\
\text { ram ciljeve i dogovaramo ono što bih trebao činiti u budućnosti. }\end{array}$ & 0.54 & & & \\
\hline & PA practices / Prakse ocjene rada & & 3.153 & 9.555 & 0.830 \\
\hline $\mathrm{q} 22$ & $\begin{array}{l}\text { My manager uses PA as an element of threat. / Moj menadžer } \\
\text { koristi se ocjenom rada kao elementom prijetnje. }\end{array}$ & 0.83 & & & \\
\hline $\mathrm{q} 21$ & $\begin{array}{l}\text { I think my manager uses PA to reward some people he likes. } \\
\text { Mislim da se moj menadžer koristi ocjenom rada kako bi } \\
\text { nagradio neke ljude koje voli. }\end{array}$ & 0.78 & & & \\
\hline $\mathrm{q} 28$ & $\begin{array}{l}\text { I think that my manager uses PA to punish persons he dislikes. } \\
\text { Mislim da se moj menadžer koristi ocjenom rada kako bi } \\
\text { kažnjavao osobe koje mu se ne sviđaju. }\end{array}$ & 0.77 & & & \\
\hline $\mathrm{q} 23$ & $\begin{array}{l}\text { I think my manager evaluates my personality, not my perfor- } \\
\text { mance. / Mislim da moj menadžer procjenjuje moju osobnost, a } \\
\text { ne moj posao. }\end{array}$ & 0.77 & & & \\
\hline \multirow[t]{2}{*}{$\mathrm{q} 24$} & $\begin{array}{l}\text { I think that people who obtain high PA score are the people who } \\
\text { can present themselves well to the manager. / Mislim da visoke } \\
\text { ocjene dobivaju oni koji se menadžeru znaju prikazati dobrima. }\end{array}$ & 0.58 & & & \\
\hline & Productivity / Produktivnost & & 2.637 & 7.991 & 0.793 \\
\hline $\mathrm{q} 47$ & $\begin{array}{l}\text { PA system planned upon reaching a consensus with my } \\
\text { superior improves my working efficiency. } \\
\text { Sustav ocjenjivanja rada kojim se planira postizanje dogovora } \\
\text { s administratorom poboljšava moju radnu učinkovitost. }\end{array}$ & 0.79 & & & \\
\hline $\mathrm{q} 48$ & $\begin{array}{l}\text { Setting realistic goals and achievable targets for my work along } \\
\text { with the company's goals and targets in my PA interview } \\
\text { improves my working efficiency. / Zadavanje realnih ciljeva i } \\
\text { ostvarivih zadataka u skladu s ciljevima i zadaćama tvrtke } \\
\text { poboljšava moju radnu učinkovitost. }\end{array}$ & 0.78 & & & \\
\hline $\mathrm{q} 46$ & $\begin{array}{l}\text { If feedback is high as a result of PA, it motivates employees } \\
\text { and increases success. / Ako je povratna informacija u skladu s } \\
\text { ocjenom rada, ona motivira zaposlenike i povećava uspjeh. }\end{array}$ & 0.75 & & & \\
\hline \multirow[t]{2}{*}{$\mathrm{q} 49$} & $\begin{array}{l}\text { As a result of PA, eliminating my failures and determining my } \\
\text { training needs in according with my deficiencies will improve } \\
\text { my business efficiency. } \\
\text { Ispravljanje mojih nedostataka kao rezultat ocjene rada i } \\
\text { određivanje mojih potreba za izobrazbom radi uklanjanja } \\
\text { mojih nedostataka poboljšava moju učinkovitost u poslovanju. }\end{array}$ & 0.61 & & & \\
\hline & Motivation / Motivacija & & 2.228 & 6.753 & 0.788 \\
\hline $\mathrm{q} 43$ & $\begin{array}{l}\text { Motivation of a high performance person will be higher. } \\
\text { Motivacija pojedinca s visokim učinkom bit će veća. }\end{array}$ & 0.80 & & & \\
\hline $\mathrm{q} 44$ & $\begin{array}{l}\text { Performance of a high motivation person will be higher. } \\
\text { Produktivnost pojedinca s visokom motivacijom bit će veća. }\end{array}$ & 0.72 & & & \\
\hline $\mathrm{q} 42$ & $\begin{array}{l}\text { There is a positive effect of PA on motivation in terms of } \\
\text { employees' self-expression, their regular communication, and } \\
\text { sharing their problems / Postoji pozitivan učinak ocjene rada } \\
\text { na motivaciju u smislu samokritičnosti zaposlenika, njihove } \\
\text { redovite komunikacije i dijeljenja njihovih problema. }\end{array}$ & 0.69 & & & \\
\hline & $\begin{array}{l}\text { Announced Total Variances, \% / Predviđena varijanca } \\
\text { Kaiser-Meyer-Olkin (KMO) value / KMO vrijednost } \\
\text { Bartlett's Test of Sphericity (Sig.) / Bartlettov test sferičnosti }\end{array}$ & - & - & $\begin{array}{c}59.942 \\
0.917 \\
0.001 \\
\end{array}$ & \\
\hline
\end{tabular}


Table 3 Reliability values of the model

Tablica 3. Pokazatelji pouzdanosti modela

\begin{tabular}{|c|c|c|c|}
\hline & $\begin{array}{l}\text { Variables } \\
\text { Varijable }\end{array}$ & $\begin{array}{c}\text { Std Loading } \\
\text { Standardna devijacija }\end{array}$ & $t^{*}$ \\
\hline $\begin{array}{l}\text { Purpose of performance appraisal } \\
\text { Cilj procjene rada } \\
(\mathrm{CR}=0.88, \mathrm{AVE}=0.50)\end{array}$ & $\begin{array}{l}\text { q3 } \\
\text { q2 } \\
\text { q1 } \\
\text { q4 } \\
\text { q5 } \\
\text { q6 } \\
\text { q7 }\end{array}$ & $\begin{array}{l}0.710 \\
0.737 \\
0.728 \\
0.726 \\
0.698 \\
0.695 \\
0.646\end{array}$ & $\begin{array}{c}14.217 \\
- \\
14.593 \\
14.554 \\
13.972 \\
13.913 \\
12.896\end{array}$ \\
\hline $\begin{array}{l}\text { Performance appraisal criteria } \\
\text { Kriteriji ocjene rada } \\
(\mathrm{CR}=0.90, \mathrm{AVE}=0.51)\end{array}$ & $\begin{array}{l}\text { q26 } \\
\text { q27 } \\
\text { q16 } \\
\text { q18 } \\
\text { q17 } \\
\text { q25 } \\
\text { q20 } \\
\text { q14 }\end{array}$ & $\begin{array}{l}0.795 \\
0.696 \\
0.729 \\
0.715 \\
0.660 \\
0.648 \\
0.661 \\
0.592 \\
\end{array}$ & $\begin{array}{c}12.754 \\
12.945 \\
- \\
14.207 \\
13.103 \\
10.851 \\
13.134 \\
11.732 \\
\end{array}$ \\
\hline $\begin{array}{l}\text { Feedback in performance appraisal } \\
\text { Povratna informacija o ocjeni rada } \\
(\mathrm{CR}=0.88, \mathrm{AVE}=0.55)\end{array}$ & $\begin{array}{l}\text { q33 } \\
\text { q32 } \\
\text { q35 } \\
\text { q36 } \\
\text { q34 } \\
\text { q40 } \\
\end{array}$ & $\begin{array}{l}0.800 \\
0.723 \\
0.725 \\
0.692 \\
0.765 \\
0.730 \\
\end{array}$ & $\begin{array}{r}- \\
15.800 \\
15.872 \\
14.991 \\
16.934 \\
16.001 \\
\end{array}$ \\
\hline $\begin{array}{l}\text { Performance appraisal practices } \\
\text { Prakse } u \text { ocjeni rada } \\
(\mathrm{CR}=0.83, \mathrm{AVE}=0.51)\end{array}$ & $\begin{array}{l}\text { q22 } \\
\text { q21 } \\
\text { q28 } \\
\text { q23 } \\
\text { q24 }\end{array}$ & $\begin{array}{l}0.847 \\
0.710 \\
0.735 \\
0.714 \\
0.502 \\
\end{array}$ & $\begin{array}{c}- \\
15.293 \\
15.924 \\
15.392 \\
10.250 \\
\end{array}$ \\
\hline $\begin{array}{l}\text { Productivity / Produktivnost } \\
(\mathrm{CR}=0.80, \mathrm{AVE}=0.50)\end{array}$ & $\begin{array}{l}\mathrm{q} 46 \\
\mathrm{q} 47 \\
\mathrm{q} 48 \\
\mathrm{q} 49\end{array}$ & $\begin{array}{l}0.710 \\
0.724 \\
0.754 \\
0.629 \\
\end{array}$ & $\begin{array}{c}12.724 \\
- \\
13.295 \\
11.463 \\
\end{array}$ \\
\hline $\begin{array}{l}\text { Motivation / Motivacija } \\
(\mathrm{CR}=0.84, \mathrm{AVE}=0.63)\end{array}$ & $\begin{array}{l}\mathrm{q} 42 \\
\mathrm{q} 43 \\
\mathrm{q} 44\end{array}$ & $\begin{array}{l}0.753 \\
0.766 \\
0.718 \\
\end{array}$ & $\begin{array}{c}- \\
13.818 \\
13.209 \\
\end{array}$ \\
\hline
\end{tabular}

* $P$ values belonging to all $t$ values is determined as 0.000 . / Vrijednosti P koje pripadaju svim vrijednostima t jednakima 0,000.

tions were significant at 0.05 significance level. Therefore, it can be concluded that the validity of the model was provided.

Two types of reliability measures were used in the measurement model: the announced variance of the factors and the reliability coefficients of the factors. While the announced variance estimates of the factors show the total variance value explained by the observed variables of each factor, the reliability coefficients of the factors show the intrinsic reliabil- ity of the factors. As shown in Tables 2 and 3, the announced variance values of the factors are above the required lower limit $(0.50 \%)$ and the reliability coefficients of the factors are again above the lower limit (0.70).

The correlations among the purpose of performance appraisal, performance appraisal criteria, feedback in performance appraisal, performance appraisal practices, productivity and motivation are presented in Table 4 along with a discriminant validity test.

Table 4 Correlation matrix for the measurement model

Tablica 4. Korelacijska matrica za model mjerenja

\begin{tabular}{|c|c|c|c|c|c|c|}
\hline $\begin{array}{c}\text { Scales } \\
\text { Ljestvice }\end{array}$ & ppa & pac & fpa & pap & $\begin{array}{c}\text { Productivity } \\
\text { Produktivnost }\end{array}$ & $\begin{array}{c}\text { Motivation } \\
\text { Motivacija }\end{array}$ \\
\hline $\mathrm{ppa}$ & 1 & & & & & \\
\hline $\mathrm{pac}$ & 0.737 & 1 & & & & \\
\hline $\mathrm{fpa}$ & 0.595 & 0.771 & 1 & & & \\
\hline $\mathrm{pap}$ & 0.253 & 0.408 & 0.336 & 1 & & \\
\hline Productivity / Produktivnost & 0.466 & 0.443 & 0.393 & 0.181 & 1 & \\
\hline Motivation / Motivacija & 0.497 & 0.562 & 0.553 & 0.134 & 0.576 & 1 \\
\hline
\end{tabular}




\subsection{Structural model}

3.4. Strukturni model

Table 5 gives the fit indexes belonging to the resulting findings as a result of the testing with the structural equation modeling analysis of the developed model.

As the fit indexes of the structural model were analyzed, it was seen that the model is within acceptable limits determined by the following literature (Anderson and Gerbing, 1984; Hancock and Mueller, 2006; Al-Refaie, 2015). AMOS program output of the model is shown in Figure 2.
Table 5 Fit indexes belonging to the results of the structural equation model

Tablica 5. Indeksi koji pripadaju rezultatima modela strukturne jednadžbe

\begin{tabular}{|l|c|c|c|c|c|c|}
\hline Model & $\boldsymbol{\chi} \mathbf{2}$ & df & $\begin{array}{c}\text { P- } \\
\text { value }\end{array}$ & $\chi \mathbf{2} / \mathbf{d f}$ & GFI & RMSEA \\
\hline $\begin{array}{l}\text { Default } \\
\text { model }\end{array}$ & 1026.554 & 480 & 0.001 & 2.139 & 0.877 & 0.051 \\
\hline
\end{tabular}

Hypothesis was examined after the validity of the model was provided. The results of hypothesis tests are given in Table 6.

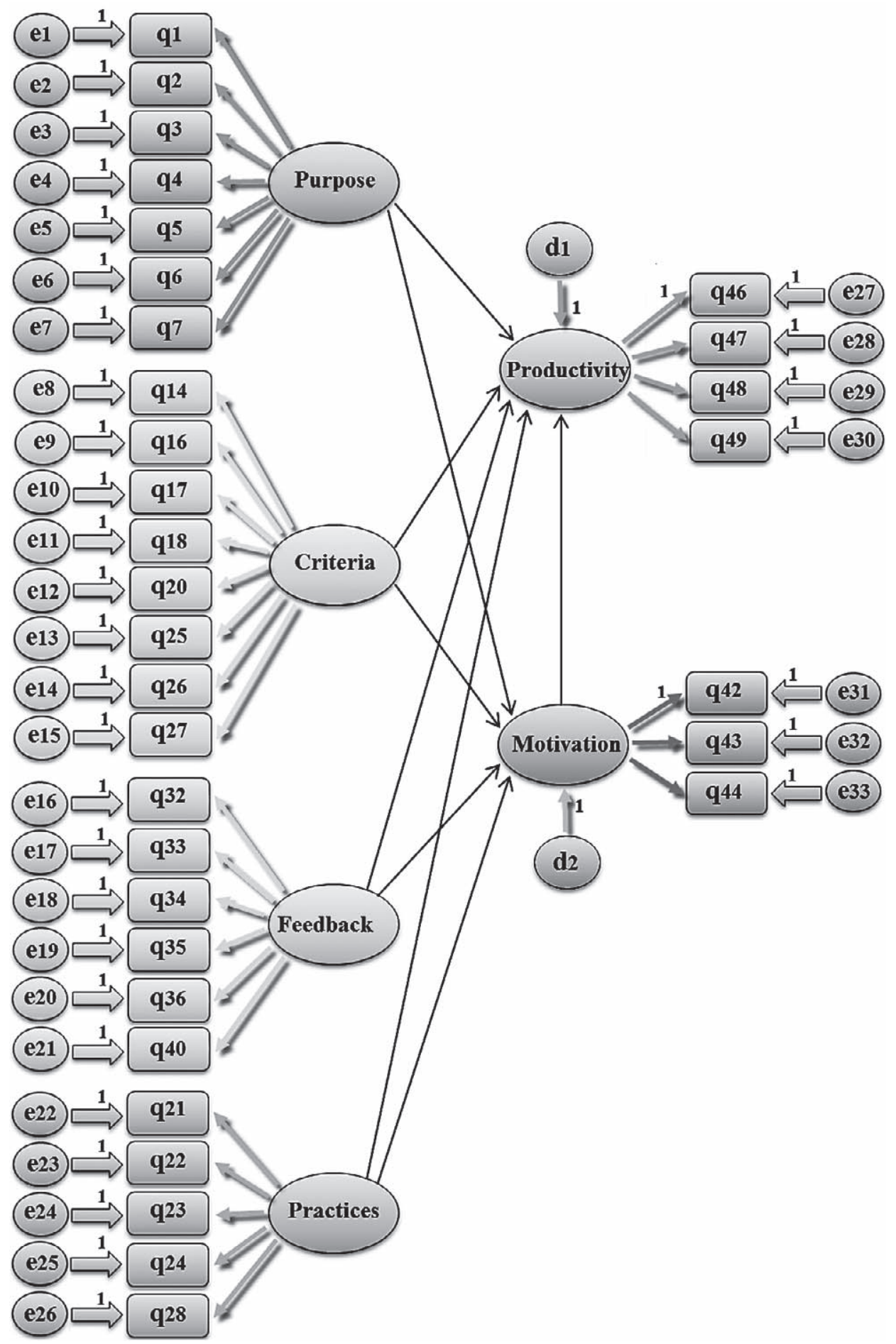

Figure 2 AMOS program output of the analyzed model Slika 2. Rezultat programa AMOS za analizirani model 
Table 6 Results of hypothesis tests

Tablica 6. Rezultati testiranja hipoteza

\begin{tabular}{|c|l|c|c|}
\hline \multicolumn{2}{|c|}{ Hypothesis / Hipoteza } & $\begin{array}{c}\text { Estimate (r) } \\
\text { Procjena (r) }\end{array}$ & $\begin{array}{c}\text { Decision } \\
\text { Odluka }\end{array}$ \\
\hline $\mathrm{H}_{1}::$ & $\begin{array}{l}\text { Purpose of PA and employee motivation } \\
\text { Svrha ocjene rada i motivacija zaposlenika }\end{array}$ & 0.156 & $\begin{array}{l}\text { Rejected } \\
\text { odbijeno }\end{array}$ \\
\hline $\mathrm{H}_{1}:$ & $\begin{array}{l}\text { Criteria of PA and employee motivation } \\
\text { Kriteriji ocjene rada i motivacija zaposlenika }\end{array}$ & $0.276^{* *}$ & $\begin{array}{l}\text { Supported } \\
\text { podržano }\end{array}$ \\
\hline $\mathrm{H}_{1} 3:$ & $\begin{array}{l}\text { Practices of PA and employee motivation } \\
\text { Prakse ocjene rada i motivacija zaposlenika }\end{array}$ & $-0.157^{*}$ & $\begin{array}{l}\text { Supported } \\
\text { podržano }\end{array}$ \\
\hline $\mathrm{H}_{1} 4:$ & $\begin{array}{l}\text { Feedback of PA and employee motivation } \\
\text { Povratne informacije o ocjeni rada i motiviranost zaposlenika }\end{array}$ & $0.278^{* *}$ & $\begin{array}{l}\text { Supported } \\
\text { podržano }\end{array}$ \\
\hline $\mathrm{H}_{1} 5:$ & $\begin{array}{l}\text { Purpose of PA and employee productivity } \\
\text { Svrha ocjene rada i produktivnost zaposlenika }\end{array}$ & $0.187^{*}$ & $\begin{array}{l}\text { Supported } \\
\text { podržano }\end{array}$ \\
\hline $\mathrm{H}_{1} 6:$ & $\begin{array}{l}\text { Criteria of PA and employee productivity } \\
\text { Kriteriji ocjene rada i produktivnost zaposlenika }\end{array}$ & 0.005 & $\begin{array}{l}\text { Rejected } \\
\text { odbijeno }\end{array}$ \\
\hline $\mathrm{H}_{1} 7:$ & $\begin{array}{l}\text { Practices of PA and employee productivity } \\
\text { Prakse ocjene rada i produktivnost zaposlenika }\end{array}$ & 0.074 & $\begin{array}{l}\text { Rejected } \\
\text { odbijeno }\end{array}$ \\
\hline $\mathrm{H}_{1} 8:$ & $\begin{array}{l}\text { Feedback of PA and employee productivity } \\
\text { Povratne informacije o ocjeni rada i produktivnost zaposlenika }\end{array}$ & -0.022 & $\begin{array}{l}\text { Rejected } \\
\text { odbijeno }\end{array}$ \\
\hline $\mathrm{H}_{1} 9:$ & $\begin{array}{l}\text { Employee motivation and employee productivity } \\
\text { Motivacija i produktivnost zaposlenika }\end{array}$ & $0.365^{*}$ & $\begin{array}{l}\text { Supported } \\
\text { podržano }\end{array}$ \\
\hline
\end{tabular}

Note: ${ }^{*} p<0.05,{ }^{*} p<0.01$

When the results of the model were analyzed, it was seen that five of the nine hypothesis $(p<0.05)$ were accepted, while four of them were rejected.

$\mathrm{H}_{1} 1$ examines the relationship between the purpose of PA and employee motivation. According to the model, there is no significant effect of the purpose of PA on employee motivation (estimate $=0.156 ; p>0.05$ ). Therefore, $\mathrm{H}_{1} 1$ hypothesis is rejected.

$\mathrm{H}_{1} 2$ examines the relationship between the criteria of PA and employee motivation. According to the model, there is a positive and significant impact of the criteria of PA on employee motivation (estimate $=0.276$; $p<0.05$ ). Therefore, $\mathrm{H}_{1} 2$ hypothesis is accepted. Accordingly, an increase of a standard unit in the criteria of PA constitutes an increase of 0.276 standard unit on employee motivation.

$\mathrm{H}_{1} 3$ examines the relationship between the practices of PA and employee motivation. According to the model, there is a negative and significant impact of the practices of $\mathrm{PA}$ on employee motivation (estimate $=-0.157 ; p<0.01$ ). Therefore, $\mathrm{H}_{1} 3$ hypothesis is accepted. Accordingly, an increase of a standard unit in the practices of PA constitutes a decrease of 0.157 standard unit on employee motivation.

$\mathrm{H}_{4} 4$ examines the relationship between the feedback of PA and employee motivation. According to the model, there is a positive and significant impact of the feedback of PA on employee motivation (estimate $=0.278 ; p<0.05$ ). Therefore, $\mathrm{H}_{4} 4$ hypothesis is accepted. Accordingly, an increase of a standard unit in the feedback of PA constitutes an increase of 0.276 standard unit on employee motivation.

$\mathrm{H}_{1} 5$ examines the relationship between the purpose of PA and employee productivity. According to the model, there is a positive and significant impact of the purpose of PA on employee productivity (estimate $=0.187 ; p<0.01$ ). Therefore, $\mathrm{H}_{1} 5$ hypothesis is ac- cepted. Accordingly, an increase of a standard unit in the purpose of PA constitutes an increase of 0.187 standard unit on employee productivity.

$\mathrm{H}_{1} 6$ examines the relationship between the criteria of PA and employee productivity. According to the model, there is no significant effect of the criteria of PA on employee productivity (estimate $=0.005 ; p<0.05$ ). Therefore, $\mathrm{H}_{1} 6$ hypothesis is rejected.

$\mathrm{H}_{1} 7$ examines the relationship between the practices of PA and employee productivity. According to the model, there is no significant effect of the practices of PA on employee productivity (estimate $=0.074$; $p<0.05$ ). Therefore, $\mathrm{H}_{1} 7$ hypothesis is rejected.

$\mathrm{H}_{1} 8$ examines the relationship between the feedback of PA and employee productivity. According to the model, there is no significant effect of the feedback of PA on employee productivity (estimate $=-0.022$; $p<0.05$ ). Therefore, $\mathrm{H}_{1} 8$ hypothesis is rejected.

$\mathrm{H}_{1} 9$ examines the relationship between employee motivation and employee productivity. According to the model, there is a positive and significant impact of employee motivation on employee productivity (estimate $=0.365 ; p<0.01$ ). Therefore, $\mathrm{H}_{1} 9$ hypothesis is accepted. Accordingly, an increase of a standard unit in employee motivation constitutes an increase of 0.365 standard unit on employee productivity.

\section{CONCLUSIONS}

\section{ZAKLJUČAK}

In this study, the effects of PA applications applied in businesses that operate in the forest products industry on employee motivation and productivity were examined by means of a structural equation model. Upon analyzing the obtained results, it could be seen that the PA had a positive impact on employee productivity, while it did not have a significant impact 
on employee motivation. There was a positive effect of the criteria of PA on employee motivation, while there was no significant impact on employee productivity. There was a negative effect of the practices of PA on employee motivation, while there was no significant impact on employee productivity. The feedback studies in PA have been found to have a positive impact on employee motivation. However, it was shown that its impact on employee productivity was not significant. Besides, it was determined that employee motivation had a positive effect on employee productivity.

In the light of these results, the following suggestions have been made for the forest products sector.

- The purpose of the employee PA should be explained to the employees very thoroughly and thus their hesitations should be reduced or avoided. It should take into consideration that an increase in the employee motivation can be provided by using PA.

- PA criteria are required so that employees can reach achievable targets. Besides, it should be explained how to help the employees to provide the criteria. It should be noted that in this way an increase in employee productivity together with employee motivation can also be provided.

- In order to achieve the purpose of the PA, in making any decision about the employees, appraisal results should be used as the data source for education, career, job rotation, pricing, etc.

- By sharing the results of the PA studies with the employees, elimination of errors and deficiencies can be provided. In-service training activities can also be organized to solve employee errors.

\section{Acknowledgement - Zahvala}

This research was supported by the Scientific Research Projects Fund of Karadeniz Technical University, Trabzon Turkey; Project no. 9760.

\section{REFERENCES}

\section{LITERATURA}

1. Al-Refaie, A., 2015: Effects of human resource management on hotel performance using structural equation modeling. Computers in Human Behavior, 43: 293-303. https://doi.org/10.1016/j.chb.2014.11.016.

2. Ali, R.; Ahmed, M. S., 2009: The Impact of reward and recognition programs on employee's motivation and satisfaction. International Review of Business Research, 5 (4): 270-279.

3. Anderson, J. C.; Gerbing, D. W., 1984: The Effect of sampling error on convergence, improper solutions and goodness of fit indices for maximum likelihood confirmatory factor analysis. Psychometrika, 9: 155-173. https://doi.org/10.1007/BF02294170.

4. Bazarganipour, F.; Ziaei, S.; Montazeri, A.; Foroozanfard, F.; Kazemnejad, A.; Faghihzadeh, S., 2013: Predictive factors of health-related quality of life in patients with polycystic ovary syndrome: a structural equation modeling approach. Mental Health, Sexuality, and Ethics, 5: 1389-1396.

https://doi.org/10.1016/j.fertnstert.2013.06.043.

5. Booth, R.; Hernandez, M.; Baker, E. L.; Grajales, T.; Pribis, P., 2013: Food safety attitudes in college students: a structural equation modeling analysis of a conceptual model. Nutrients, 5: 328-339.

https://doi.org/10.3390/nu5020328.

6. Chan, F.; Lee, G. K.; Kubota, C.; Allen, C. A., 2007: Structural equation modeling in rehabilitation counseling research. Rehabilitation Counseling Bulletin, 51 (1): 5366. https://doi.org/10.1177/00343552070510010701.

7. Chang, E.; Hahn, J., 2006: Does pay-for-performance enhance perceived distributive justice for collectivistic employees? Personnel Review, 35 (4): 397-412.

https://doi.org/10.1108/00483480610670571.

8. Dunteman, G. H., 1989: Principal components analysis. Quantitative applications in the social sciences series, No. 69. Thousand Oaks, CA: Sage publications.

9. Fakhimi, F.; Raisy, A., 2013: Satisfaction with performance appraisal from the employees' perspective and its behavioral outcomes (case study of headquarters offices of Bank Refah). European Online Journal of Natural and Social Sciences, 2 (3): 296-305.

10. Gichuhi, A. W.; Abaja, P. O.; Ochieng, I., 2013: Effect of performance appraisal on employee productivity; a case study of supermarkets in Nakuru Town, Kenya. Asian Journal of Business and Management Sciences, 2 (11): $42-58$.

11. Gürbüz, S.; Dikmenli, O., 2007: Performance appraisal biases in a public organization: an emprical study, Kocaeli Üniversitesi Sosyal Bilimler Enstitüsü Dergisi, KOSBED 13 (1): 108-138.

12. Hallak, R.; Brown, G.; Lindsay, N. J., 2012: The Place Identity of Performance relationship among tourism entrepreneurs: A structural equation modelling analysis. Tourism Management, 33: 143-154. https://doi.org/10.1177/0047287513513170.

13. Hancock, G. R.; Mueller, R. O., 2006: Structural equation modeling: a second course, Greenwich, CT: Information Age Publishing, Inc., 432 p.

14. Hitka, M.; Stipalova, L., 2011: Comparing of employees motivation level in enterprises of wood working industry with other manufacturing enterprises in Slovak Republic. Drvna industrija, 62 (3): 185-192. https://doi.org/10.5552/drind.2011.1101.

15. Hitka, M.; Hajdukova, A.; Balazova, Z., 2014: Impact of economic crisis on changes in motivation of employees in woodworking industry. Drvna Industrija, 65 (1): 2126. https://doi.org/10.5552/drind.2014.1303.

16. Hsu, I. Y.; Su, T. S.; Kao, C. S.; Shu, Y. L.; Lin, P. L.; Tseng, J. M., 2012: Analysis of business safety performance by structural equation models. Safety Science, 50 : 1-11. https://doi.org/10.1016/j.ssci.2011.04.012.

17. Jelačić, D.; Grladinović, T.; Sujova, A.; Galajdova, V., 2008: Motivation factors in wood processing and furniture manufacturing. Drvna industrija, 59 (1): 11-21.

18. Kalayc1, S., 2009: SPSS Applied Multivariate Statistical Techniques. Asil Release Distribution, Ankara, 426 p.

19. Kropivšek, J.; Jelačić, D.; Grošelj, P., 2011: Motivating employees of Slovenian and Croatian wood-industry companies in times of economic downturn. Drvna industrija, 62 (2): 97-103. https://doi.org/10.5552/drind.2011.1040.

20. Kumbhar, S. A., 2011: Impact of performance appraisal on corporate profitability, Indian Streams Research Journal.

21. Lorincova, S.; Schmidtova, J.; Javorcíkova, J., 2016: Employee job satisfaction in furniture manufacturing companies in the Slovak Republic. Drvna industrija, 67 (4): 351-362. https://doi.org/10.5552/drind.2016.1614.

22. Ochoti, G. N.; Maronga, E.; Muathe, S.; Nyabwanga, R. N.; Ronoh, P. K., 2012: Factors influencing employee 
performance appraisal system: a case of the ministry of state for provincial administration \& internal security, Kenya. International Journal of Business and Social Science, 3(2): 38-46.

23. Osibanjo, A.; Salau, O.; Falola, H., 2014: Modeling the relationship between motivating factors; employee's retention; and job satisfaction in the Nigerian banking 1ndustry. Journal of Management Policies and Practices, 2 (2): 63-83.

24. Phin, L. W., 2015: The effectiveness of performance appraisal in the private education industry in Malaysia. International Journal of Business and Information, 10 (1): 95-124.

25. Prowse, P.; Prowse, J., 2009: The dilemma of performance appraisal. Journal of Measuring Business Excellence, 13 (4): 69-77. https://doi.org/10.1108/13683040911006800

26. Salau, O. P.; Oludayo, O. F.; Omoniyi, O. C.; Akinbode, J. O., 2014: Modelling the relationship between performance appraisal and organizational productivity in Nigerian public sector. Economics Management Innovation, 6 (2): $1-16$.

27. Serin, H., 2004: Total quality management of furniture industry establishment in the organized industrial zones in Turkey, Ph.D. Thesis, Karadeniz Technical University, Institute of Science, Trabzon, Turkey.

28. Sharma, S., 1996: Applied multivariate techniques. John Wiley \& Sons Inc., New York.

29. Singh, V. K.; Kochar, B.; Yüksel, S., 2010: An empirical study on the efficiency of performance appraisal system in oil and natural gas commission (ONGC), India, Journal of Business Research-Turk, 2: 65-78.

30. Suki, N. M., 2014: Passenger satisfaction with airline service quality in Malaysia: A structural equation modeling approach. Research in Transportation Business \& Management, 10: 26-32. https://doi.org/10.1016/j.rtbm.2014.04.001.
31. Tarlı̆ , Y. T., 2006: An investigation on identifying employee attitudes for performance evaluation process and areas in which performance evaluation results are applied. Master Thesis, Yıldız Technical University Social Sciences Institute, İstanbul, Turkey.

32. Vasset, F.; Marnburg, E.; Furunes, T., 2011: The effects of performance appraisal in the Norwegian municipal health services: a case study, Human Resources for Health, 9 (22): 1-12. https://doi.org/10.1186/1478-4491-9-22

33. Wholey, J., 1999: Performance based management: Responding to the challenges, Public Productivity and Management Review, 22: 288-307. https://doi.org/10.2307/3380705.

34. Xiong, B.; Skitmore, M.; Xia, B., 2015: A critical review of structural equation modeling applications in construction research, Automation in Construction, 49: 59-70. https://doi.org/10.1016/j.autcon.2014.09.006.

35. Xiong, B.; Skitmore, M.; Xia, B.; Masrom, M. A.; Ye, K.; Bridge, A., 2014: Examining the influence of participant performance factors on contractor satisfaction: A structural equation model. International Journal of Project Management, 32: 482-491. https://doi.org/10.1016/j.ijproman.2013.06.003.

36. Yilmaz, E. F., 2006: The effect of the performance evolution system on the productivity of administrating and a case study. Master Thesis, Trakya University, Social Sciences Institute. Edirne, Turkey.

\section{Corresponding address:}

\section{Assist. Prof. AYTAÇ AYDIN}

Department of Forest Industry Engineering Faculty of Forestry, Karadeniz Technical University 61080 Trabzon, TURKEY

e-mail: aytac@ktu.edu.tr 